\title{
Fungal gasdermin-like proteins are controlled by proteolytic cleavage
}

\author{
Corinne Clavéa, Witold Dyrkac, Alexandra Granger-Farbos ${ }^{a}$, Benoît Pinson ${ }^{b}$, Sven J.
}

Saupe a and Asen Daskalov ${ }^{\mathrm{a}}$

aNon-self Recognition in Fungi, Institut de Biochimie et Génétique Cellulaires, UMR 5095 CNRS, Université de Bordeaux, 1 Rue Camille Saint Saëns, 33077

\author{
Bordeaux CEDEX, France
}

${ }^{\mathrm{b}}$ Genetics of Metabolic Pathways, Institut de Biochimie et Génétique Cellulaires, UMR 5095 CNRS, Université de Bordeaux, 1 Rue Camille Saint Saëns, 33077

\author{
Bordeaux CEDEX, France
}

cPolitechnika Wrocławska, Wydział Podstawowych Problemów Techniki, Katedra Inżynierii Biomedycznej, Wybrzeże Wyspiańskiego 27,

50-370 Wrocław, Poland 


\section{Corresponding authors}

CNRS, UMR 5095, Institut de Biochimie et Génétique Cellulaires, University of Bordeaux,

CNRS, UMR 5095, Institut de Biochimie et Génétique Cellulaires, University of Bordeaux, 
54

\section{Abstract}

Gasdermins are a family of pore-forming proteins controlling an inflammatory cell death reaction in the mammalian immune system. The pore-forming ability of the gasdermin proteins is released by proteolytic cleavage with the removal of their inhibitory C-terminal domain. Recently, gasdermin-like proteins have been discovered in fungi and characterized as cell deathinducing toxins in the context of conspecific non-self discrimination (allorecognition). Although functional analogies have been established between mammalian and fungal gasdermins, the molecular pathways regulating gasdermin activity in fungi remain largely unknown. Here, we characterize a gasdermin-based cell death reaction, controlled by the het- $Q$ allorecognition genes in the filamentous fungus Podospora anserina. We show that the cytotoxic activity of the HET-Q1 gasdermin is controlled by proteolysis. HET-Q1 loses a $\sim 5 \mathrm{kDa}$ C-terminal fragment during the cell death reaction in presence of a subtilisin-like serine protease, termed HET-Q2. Mutational analyses and successful reconstitution of the cell death reaction in a heterologous host (Saccharomyces cerevisiae) suggest that HET-Q2 directly cleaves HET-Q1 to induce cell death. By analysing the genomic landscape of het-Q1 homologs in fungi, we uncovered that the vast majority of the gasdermin genes are clustered with protease-encoding genes. These HETQ2-like proteins carry either subtilisin-like or caspase-related proteases, which in some cases correspond to the N-terminal effector domain of NOD-like receptor proteins (NLRs). This study thus reveals the proteolytic regulation of gasdermins in fungi and establishes evolutionary parallels between fungal and mammalian gasdermin-dependent cell death pathways.

\section{Keywords}

Gasdermin, Innate Immunity, Regulated Cell Death, Proteolysis, Podospora, Fungi

\section{Significance}

The recent discovery of gasdermin-like proteins in fungi have brought to light that this family of pore-forming proteins controls cell death in two of the major eukaryotic kingdoms, fungi and mammals. Yet, the regulation of cytotoxicity of the fungal gasdermins and their molecular pathways remain uncharacterized. Here, we describe the regulation through proteolytic cleavage of the fungal gasdermin HET-Q1 and uncover that majority of fungal gasdermins are genomically clustered with protease-encoding genes. Some of these genes encode proteins with caspase-related domains and/or are members of a family of immune receptors in mammals and plants. Overall, this work contributes towards our understanding of the evolution of gasdermindependent cell death, enlightening multiple evolutionary parallels between signaling pathways in mammals and fungi. 


\section{Introduction}

Spatiotemporally localized, regulated cell death (RCD) prevents cytoplasmic mixing and heterokaryon formation between conspecific individuals in fungi $(1,2)$. The cell death reaction limits the transmission of mycoviruses and other deleterious replicons, thus representing a defence reaction akin to an immune response $(3,4)$. The molecular characterization of cell death-pathways, regulating conspecific non-self discrimination (allorecognition) in fungi, has revealed evolutionary relations to cell death pathways operating in mammalian innate immunity, notably necroptosis and pyroptosis $(5,6)$. The extent of conservation of these pathways is gradually revealed as the characterization of fungal RCD pathways progresses.

Proteolytic cleavage is a general mechanism for regulation of programmed cell death (PCD) and RCD in metazoans and plants (7). In mammals, a family of cysteine-aspartic proteases (caspases) controls cellular suicide pathways like apoptosis and pyroptosis $(8,9)$. The latter represents a highly inflammatory, lytic cell death reaction, playing a central role in mammalian innate immunity $(10,11)$. Pyroptotic cell death is induced by pro-inflammatory caspases (caspase-1, $-4,-5$ and -11 ), which cleave a protein termed gasdermin D (GSDMD) (12, 13). The cleavage of GSDMD liberates the cytotoxic N-terminal domain of the protein (GSDMDNT) from the inhibitory C-terminal domain (GSDMD-CT) and the processed GSDMD-NT disrupts the integrity of the plasma membrane by pore-formation (14-17). GSDMD belongs to a protein family comprising six members in humans (GSDMA, GSDMB, GSDMC, GSDME (also DFNA5) and PJVK (also DFNB59)) (18). Proteolytic cleavage has been shown to regulate the cytotoxic activity of other members of the gasdermin family, specifically of GSDME (19) and GSDMB (20). While the regulation of GSDME is caspase-3-dependent $(19,21)$, it has recently been reported that GSDMB is proteolytically activated by serine protease termed granzyme A (GZMA) (20).

Gasdermin-like proteins are abundant and widespread in fungi with some species encoding more than twenty gasdermin-homologs in their genomes (22). It has recently been established that a fungal gasdermin-like protein controls cell death in the context of allorecognition. The co-expression of antagonistic alleles of the $r c d-1$ gene (regulator of cell death) triggers rapid, lytic cell death in asexual spores and hyphae of the model ascomycete Neurospora crassa (22). RCD-1 is a distant homolog of the cytotoxic pore-forming GSDMD-NT domain and, like gasdermin, induces cell death by targeting the plasma membrane, while forming oligomers (6). However, in spite of the uncovered functional similarities between the mammalian gasdermins and fungal RCD-gasdermin-like protein, in the latter case, cytotoxic activity is not dependent on proteolytic activation but results instead from the interaction of antagonistic RCD-variants. 
Here, we characterized the fungal gasdermin het-Q1 form Podospora anserina and report that its cytotoxic activity is regulated through proteolytic cleavage by a subtilisin-like serine protease named HET-Q2. het-Q1 and het-Q2 are idiomorphic genes (they represent alternate alleles of the same het- $Q$ locus but are totally unrelated in sequence) and regulate cell death in the context of heterokaryon formation (somatic fusions between different strains). We demonstrated that a $\sim 5 \mathrm{kDa}$ C-terminal fragment is cleaved from HET-Q1 in presence of HETQ2. Expression of a truncated variant - HET-Q1(1-233) - was sufficient to reduce cell viability, while a point mutant - HET-Q1-F238A - preventing proteolytic cleavage of the fungal gasdermin, abolished its cytotoxicity. Catalytically inactive mutants of the HET-Q2 protease were equally unable to produce cell death when co-expressed with HET-Q1. The het-Q1/het-Q2 RCD reaction can be reconstructed in the distant heterologous yeast host Saccharomyces cerevisiae, which strongly suggests that HET-Q2 removes directly the C-terminal inhibitory end of the gasdermin HET-Q1 to induce cell death. Importantly, in silico analyses of the genomic landscape of het-Q1 homologs revealed that the fungal gasdermin genes are, in the vast majority of cases, the genomic neighbours of protease-encoding het-Q2-like genes. In addition, $\sim 20 \%$ of these protease-encoding neighbours carry a predicted caspase-like domain. Our work indicating that members of the gasdermin family in fungi are regulated through proteolytic cleavage reveals a remarkable evolutionary conservation of regulated cell death pathways in Eukaryotes.

Results

\section{RCD during het- $Q$-allorecognition is controlled by idiomorphic genes}

The het- $Q$ locus is one of the last uncharacterized allorecognition loci in P. anserina (23). $P$. anserina strains either belong to the het-Q1 or het-Q2 genotype. Cellular fusions between strains from different het- $Q$ genotypes are abortive and trigger RCD, which translates with the appearance of a 'barrage' (demarcation line) between such strains. The het- $Q$ locus has been genetically located on chromosome 7 (24). To identify the precise genetic determinants controlling het- $Q$ RCD, we analyzed the genetic distance between het- $Q$ and the PaATG1 locus (Pa_7_10890), situated on the same chromosome (25). We measured $\sim 2.5 \%$ of recombination between het- $Q$ and PaATG1, which corresponds to $\sim 60 \mathrm{kbp}$ in estimated physical distance between the two genes. We thus undertook a positional cloning approach with a DNA library of cosmids and plasmids from a het-Q1 strain, covering a region of $\sim 100 \mathrm{kbp}$ around PaATG1 gene (SI Appendix, Fig. S1, Tables S1, S2, S3). Cosmid and plasmid clones were transformed into protoplasts of the incompatible het-Q2 genetic background. Since co-expression of het-Q1 and het-Q2 is lethal, it is expected that clones bearing het-Q1 show reduced transformation efficiency in this background (SI Appendix, Fig. S1; Table S1 and S2). Only constructs bearing the 
background (SI Appendix, Fig. S1, Table S4). These experiments suggest that Pa_7_10775 is allelic to het-Q1. We replaced the Pa_7_10775 gene with a nat1 cassette (nourseothricin resistance marker) producing a $\triangle P a_{-} 7_{-} 10775$ strain, which showed wild-type growth and fertility. The $\Delta P a_{-}$7_10775 strain no longer produced a barrage reaction with a het-Q2 strain, indicating that the RCD reaction is abolished (Fig. 1A). Ectopic re-introduction of Pa_7_10775 into the $\Delta P a_{-} 7 \_10775$ background restored the barrage reaction to het-Q2 (Fig. $1 \mathrm{~A}$ and $1 \mathrm{~B}$ ). These results indicate that Pa_7_10775 is het-Q1. The $\Delta P a_{-}$7_10775 strain was thus designated Ahet-Q.

Next, we amplified the same locus from a het-Q2 strain using primers located in the ORFs flanking het-Q1 (Pa_7_10775), Pa_7_10770 and Pa_7_10780 (SI Appendix, Fig. S1). The corresponding PCR fragment showed reduced transformation efficiency in the het-Q1 background, while transformation efficiencies into the het-Q2 or $\Delta$ het- $Q$ backgrounds were at levels similar to that with the empty vector (SI Appendix, Fig. S1). $\Delta$ het-Q strains transformed with this PCR fragment acquired the het-Q2 phenotype and triggered RCD with a het-Q1 tester strain (Fig. 1). The het-Q locus situated between the Pa_7_10770 and Pa_7_10780 ORFs is totally dissimilar between the het-Q1 and het-Q2 strains and varies in length in the two backgrounds with $1.6 \mathrm{kbp}$ and $2.2 \mathrm{kbp}$, respectively (Fig. 1C). The het-Q1 and het-Q2 genes are thus best described as idiomorphs rather than classical alleles (26).

\section{het-Q1 encodes a gasdermin homolog and het-Q2 a putative serine protease}

The het-Q1 gene encodes a 278 amino acid long protein, HET-Q1, which belongs to the same protein family as RCD-1 from N. crassa (6) and thus is a fungal gasdermin-like protein (SI Appendix, Fig. S2). We confirmed the homology between HET-Q1 and mammalian gasdermins, using the HHPred suite (27). The in silico analyses uncovered two regions of higher sequence conservation between HET-Q1, GSDMD-NT and GSDMA3-NT, detected around residues 148-155 and 210-217, as previously reported for RCD-1 protein (SI Appendix, Fig. S2).

The het-Q2 ORF encodes a 366 amino acid long protein. HET-Q2 shows homology to subtilisin-like serine proteases of the S8 family (SI Appendix, Fig. S2). No signal peptide was detected in HET-Q2, which suggests that the predicted protease is not secreted. Based on sequence alignments and homology modeling, the amino acid residues D35, H105 and S266 of HET-Q2 were identified as constituting the catalytic triad of the putative protease. HET-Q2 lacks an N-terminal propeptide inhibitor domain (i.e. 19 domain), typically found in S8 subtilisin proteases (28).

\section{HET-Q2-dependent proteolytic cleavage of HET-Q1 triggers cell death}


Considering the homology with mammalian gasdermins and the fact that these cell death-inducing proteins are activated by proteolytic cleavage, we reasoned that the HETQ1/HET-Q2 cell death reaction might result from the proteolytic cleavage of the HET-Q1 gasdermin by the HET-Q2 serine protease. To test this hypothesis, we produced N-terminally V5-tagged HET-Q1 allelic variant (referred as V5-HET-Q1 or V5-Q1), which was functional and induced cell death at wild type levels (SI Appendix, Fig. S3). We then explored the proteolytic reaction by mixing crude cell extracts (cytoplasmic content) of strains expressing V5-tagged HET-Q1 with extracts from a het-Q2 strain or from the $\Delta$ het- $Q$ strain. Lysates mixtures 1 :1 ratio) were incubated for up to 30 minutes $\left(25^{\circ} \mathrm{C}\right)$. At the initial time point (at $0^{\prime}$ minutes) in both reaction mixtures $(\mathrm{V} 5-\mathrm{Q} 1+\mathrm{Q} 2$ and $\mathrm{V} 5-\mathrm{Q} 1+\Delta \mathrm{Q})$ the $\mathrm{V} 5$-tagged HET-Q1 protein was detected by Western blot at the expected molecular weight ( $\sim 35 \mathrm{kDa}$ ) (Fig. $2 \mathrm{~A})$. The molecular weight of HET-Q1 remained unchanged in the mixture of crude lysates from the v5-het-Q1 and $\Delta$ het-Q strains (V5-Q1+ $\mathrm{Q}$ ) (Fig. 2A). However, in presence of HET-Q2 (V5-Q1+Q2 mixture) we observed a progressive decrease in the amount of full-length HET-Q1 with a correlated appearance of a fragment of lower molecular weight ( 28-30 kDa), corresponding to the $\mathrm{N}$ terminal part of the HET-Q1 protein (Fig. 2A). We concluded that in presence of the HET-Q2 serine protease, HET-Q1 is proteolytically processed and a $\sim 5 \mathrm{kDa}$ C-terminal fragment (or domain) is cleaved from the protein.

To investigate whether the proteolytic cleavage activates the cytotoxicity of HET-Q1, we produced four truncated protein variants, removing short fragments in $\sim 15$ amino acids increments from the C-terminal end of the protein. The truncations were introduced in the $\Delta$ het$Q$ strain and the number of viable transformants was quantified (Fig. 2B). Three of the tested truncations (HET-Q1(1-261), HET-Q1(1-248) and HET-Q1(1-218)) showed normal levels of transformation efficiency, while the fourth truncation - HET-Q1(1-233) - resulted in significant decrease in the number of viable transformants (Fig. 2B). Western blot analyses confirmed that the HET-Q1(1-233) truncation is of similar molecular weight to processed HET-Q1 (Fig. 2C). Noteworthy, among the truncation constructs, only strains expressing HET-Q1(1-261) produce barrage with a HET-Q2-expressing strain (SI Appendix, Fig. S3). These results suggest that some of the truncations impact the N-terminal cytotoxic domain of HET-Q1 and/or prevent the activation of the toxin by the protease. We concluded that the removal of the last 45 amino acids residues of HET-Q1 leads to an increased cytotoxicity of HET-Q1. In addition, we performed size-exclusion chromatography with cellular extracts. While unprocessed HET-Q1 elutes in low molecular weight fractions, we found that in mixtures containing HET-Q1 and HET-Q2 (Q1+Q2) proteolytically processed HET-Q1 elutes in the void fraction. This result suggests that the 
proteolytic cleavage induces a transition from monomeric towards multimeric state of the fungal toxin, similarly to members of the gasdermin family in mammals (Fig. 2D) (18).

Next, we undertook to identify amino acid residues important for the proteolytic cleavage of HET-Q1 and replaced individual residues with alanines in the region where the cleavage occurs. We generated a set of seven HET-Q1 mutants, encompassing the region from residues G234 to G241 of HET-Q1 (Fig. 2E) and tested the ability of the mutants to induce cell death with a strain expressing HET-Q2 (Fig. 2F). The majority of HET-Q1 mutants were not affected in their ability to induce cell death. Only strains expressing HET-Q1-L237A and HET-Q1-F238A were unable to produce barrage with the het-Q2 strain (Fig. 2F). However, after prolonged contact between transformants expressing HET-Q1-L237A and the het-Q2 strain, we observed the formation of attenuated barrages, suggesting that the mutation affects the cell death reaction only partially. We then probed whether the proteolytic cleavage of the HET-Q1-L237A and HETQ1-F238A mutants occurs in presence of HET-Q2 (Fig. 2G). Crude cellular extracts from transformants expressing HET-Q1 mutant variants were mixed in a 1:1 ratio with cellular extracts from a $\Delta$ het- $Q$ strain or het-Q2 strain and incubated for up to 30 minutes. Mutations affecting the cell death reaction (L237A and F238A) were found to equally affect the proteolytic cleavage of HET-Q1. The two mutant variants (HET-Q1-L237A and HET-Q1-F238A) remained unprocessed even after 30 minutes in presence of HET-Q2 (Fig. 2G). Mutant HET-Q1 variants that did not affect the cell death reaction (V236A and G240A) did not abolish the proteolytic cleavage of HET-Q1 and were processed after 30 minutes in presence of HET-Q2 (Fig. 2G). We concluded from these experiments that the amino acid residues L237 and F238 are important for the proteolytic cleavage of HET-Q1 and likely integral to the cleavage site. The results further confirm that proteolysis of HET-Q1 is necessary for the induction of cytotoxicity.

\section{Proteolytic activity of the subtilisin-like protease HET-Q2 is essential for gasdermin cytotoxicity}

We reasoned that HET-Q2 might be constitutively active (or alternatively be specifically activated by the interaction with HET-Q1) and that its proteolytic activity controls the cell death reaction, likely by direct cleavage of HET-Q1. We therefore generated three het-Q2 mutants, replacing each of the residues forming the catalytic triad of HET-Q2 (D35, H105 and S266) with an alanine amino acid residue. The mutant alleles (het-Q2-D35A, het-Q2-H105A and het-Q2$S 226 A$ ) were introduced into $\Delta$ het- $Q$ background and transformants were tested for barrage formation with the het-Q1 tester strain. While $\sim 76 \%$ of transformants with wild-type het-Q2 produced a barrage reaction with the het-Q1 strain, transformants with mutant het-Q2 alleles showed reduced or abolished cell death-inducing activity (Fig. 3A). Strains transformed with two of the mutants - het-Q2-H105A and het-Q2-S226A - were unable to trigger cell death with a 
het-Q1 strain, while only $\sim 37 \%$ of transformants with the het-Q2-D35A allele produced a barrage reaction (Fig. 3A). The decrease of activity shown by het-Q2-D35A was statistically significant and consistent with previously reported residual protease activity for an equivalent mutation in a human subtilisin (29). These results demonstrate that the proteolytic activity of HET-Q2 is necessary for the control of the HET-Q1-dependent cell death reaction.

Next, we decided to use Saccharomyces cerevisiae as a heterologous system, in which to reconstitute the HET-Q1/HET-Q2 cell death reaction. We reasoned that cell death signaling pathways between the yeast and Podospora are unlikely to be conserved, as the two fungal species are phylogenetically very distant and differ in cellular organization (unicellular versus multicellular). None of the known components of $P$. anserina allorecognition pathways has homologs in yeast. We co-expressed HET-Q1 and HET-Q2 in yeast. The het-Q1 gene was under the control of a constitutive promoter, while het-Q2 was under a galactose-inducible gal1 promoter. Experiments were performed at $30^{\circ} \mathrm{C}$, the optimal growth temperatures for Saccharomyces. We found that co-expression of HET-Q1 and HET-Q2 strongly affected the growth of S. cerevisiae (Fig. 3B). Expression of HET-Q1 alone or HET-Q2 alone did not alter the growth of S. cerevisiae nor did the HET-Q2-D35A mutant when co-expressed with HET-Q1 (Fig. $3)$. Taken together, these results suggest that het-Q1 and het-Q2 genes constitute an autonomous cell death induction system and further support the direct cleavage of the HET-Q1 gasdermin by the HET-Q2 serine protease.

\section{Genomic clustering of het-Q1 homologs with protease-encoding het-Q2-like genes in fungi}

Gasdermin-encoding het-Q1 homologs are ubiquitous in Ascomycota and have been identified in more than 180 fungal species $(6,22)$. The uncovered functional relation between HET-Q1 and the HET-Q2 protease, as well as the previously reported genomic clustering of regulation and cell death execution modules in fungal necroptosis-like pathways $(5,30)$, prompted us to analyze the local genomic landscape of het-Q1 homologs in fungi. We retrieved 1884 het-Q1 homologs situated in the genomes of 401 fungal species using $P$. anserina het-Q1 as a query sequence. We found that $80 \%$ of the het-Q1 genes had at least one gene encoding for a protein with a protease domain as close neighbor (within 10-kbp) (Fig. 4A and 4B, SI Appendix, Table S5). Such genes were frequently adjacent to the gasdermin-encoding het-Q1 homologs forming two-gene clusters - and were termed het-Q2-like (Fig. 4A). Approximately 63\% (1187) of the analyzed 1884 fungal gasdermins were clustered with het-Q2-like genes encoding proteins with a putative S8 serine protease domain, as found in HET-Q2 (Fig. 4B). Remarkably, another 19\% (353) of the het-Q1 homologs were in the vicinity of a het-Q2-like gene encoding for a protein with a predicted protease domain of the CHAT (Caspase HetF Associated with TPRs) clade, which belongs to the same protease family as the mammalian caspases (31). We 
also found that $20 \%$ (373) of the het-Q1 homologs were not situated near a het-Q2-like gene, yet the vast majority of het-Q1 genes in this category (76\%) were from fungal genomes containing at least one het-Q1/het-Q2-like two-gene cluster (SI Appendix, Table S5). Overall, we identified 1617 het-Q1/het-Q2-like clusters (1417het-Q1/het-Q2-like gene pairs and 88 het-Q1/two hetQ2-like three-gene clusters) from 366 fungal species with a dozen of species carrying at least ten such clusters in their genomes (SI Appendix, Table S5). Noteworthy, we also found two distant het-Q1 homologs (Pa_6_6615 and Pa_5_3510) in the genome of P. anserina, clustered with hetQ2-like genes, one being of the S8 type, the other of the CHAT-type (SI Appendix, Fig. S4, Table S5).

The fungal gasdermins show a considerable phylogenetic diversity and formed more than 20 well-supported clades (support values above 0.9 for majority of non-singleton branches) comprising between ten and 300 het-Q1 members (Fig. 4D). Although exception occurred, each clade or super-clade was predominantly associated with either the S8 and CHAT-protease type (Fig. 4D). This observation underscores further the functional link between the clustered genes and suggests that gasdermin and protease encoding-genes spend considerable evolutionary time in association while occasional re-association with a distinct protease-type can occur.

To gain insight into the functional role of these cell death pathways, we analyzed the domain architectures of the HET-Q2-like proteins (Fig. 4C, SI Appendix, Table S6). In the set of 1601 HET-Q2-like protein sequences, 1435 (90\%) carry N- (1374) or C-termini (125) longer than 100 amino acids, in addition to the S8/CHAT protease. Using Pfam profiles, we annotated 493 of the 1499 long $\mathrm{N}$ - and C-termini (33\%). The vast majority of the annotated sequences (above 95\%) belonged to 16 Pfam clans and resulted in 18 potential protein architectures of HET-Q2-like proteins (Fig. 4C, SI Appendix, Table S6). Several of the most abundant HET-Q2-like protein architectures presented annotated domains consisting of tandem-repeat motifs (TPR, ANK or WD40 repeats), which are known to mediate protein-protein interactions and/or ligand binding functions (32) (Fig. 4C). Furthermore, we found that some of the het-Q2-like genes encode for NOD-like receptors (NLRs), exhibiting a typical tripartite domain organization with a central P-loop containing NOD (Nucleotide-binding and oligomerization) domain flanked by the protease domain and tandem-repeat motifs (specifically S8 / NACHT / WD40) (Fig. 4C). Overall, our analyses indicate that the majority of fungal gasdermins are clustered with genes encoding for putative CHAT or S8 protease domain-containing, multi-domain proteins, some of which belong to a class of cell death-controlling innate immunity receptors.

Based on these findings, we postulate that fungal gasdermins are in general regulated by proteolytic cleavage by HET-Q2-like functional partners (encoded by a closely linked gene) and are an essential part of broader, diverse and widespread signaling pathways in fungi (Fig. 4E). 
The genomic clustering of the gasdermin genes and the gene encoding their putative cognate protease is highly reminiscent of the clustering of HeLo-domain cell death execution proteins with their cognate regulatory amyloid signaling NLR $(30,33)$. In this context it is noteworthy, that the number of gasdermin homologs and Helo-domain pore-forming toxin homologs in fungal genomes are correlated (Pearson's $r=0.65$, p-value $<1 \mathrm{e}-55$, Spearman's $\rho=0.49$, $p$-value $<$ 1e-27) (SI Appendix, Table S7), suggesting that the life-style of certain species calls for a high number of parallel RCD pathways.

\section{Discussion}

In this study, by molecularly characterizing a fungal allorecognition system from Podospora anserina, we show that fungal gasdermin-like proteins are controlled by proteolytic cleavage, similar to their mammalian counterparts $(13,14)$. We uncovered that the fungal gasdermin HET-Q1 is proteolytically processed in the presence of the serine protease HET-Q2, during the allorecognition process in $P$. anserina. The proteolytic cleavage removes a $\sim 5 \mathrm{kDa} C$ terminal fragment, which unleashes the cytotoxic activity of HET-Q1. We reconstituted the cell death reaction by co-expressing HET-Q1 and HET-Q2 in the yeast Saccharomyces cerevisiae. The latter finding strongly suggests that the fungal gasdermin is directly processed by the serine protease and establishes HET-Q1/HET-Q2 protein pair as an autonomous RCD system. In this context, we uncovered by genome mining that $\sim 80 \%$ of the gasdermin-encoding genes in fungi are clustered with het-Q2-like genes, encoding proteins with a putative protease domain, which belongs in the majority of cases to the subtilisin-like serine proteases (S8 family). Our analyses also identified that a subset of the HET-Q2-like proteins carry a caspase-related CHAT domain, while others exhibit NLR-like protein architectures. These findings prompted us to propose that similarly to HET-Q1, proteolytic cleavage is the general mode of activation for the fungal gasdermins. Furthermore, we propose that for the vast majority of HET-Q1 homologs the proteolysis occurs via an activated HET-Q2-like protein, encoded by the gene adjacent to the gasdermin-encoding gene. Genomic clustering of genes encoding pore-forming toxins and the receptors that activate them has been previously reported in fungi in the case of the HeLodomain pore forming toxins and their cognate NLR receptors $(30,33)$.

\section{Gasdermin-regulated cell death in the context of comparative immunology}

This study extends the evolutionary parallels between fungal and mammalian cell death pathways beyond the previously uncovered conservation of cell death execution modules (gasdermin pore-forming domain). Our findings establish a broader evolutionary framework for gasdermin-regulated cell death by unveiling a common mode of activation (proteolysis) for the gasdermin family in Eukaryotes, performed by similar type of proteases, which can be 
associated - at least in some cases - to homologous upstream receptors (NLR proteins) (Fig. $4 \mathrm{E})$.

During pyroptosis the mammalian gasdermins are processed either by caspases (11-13, 34 ) or in the case of GSDMB by a serine protease (GZMA) (20). Serine proteases play a key role in human immunity, where they can control inflammatory and non-inflammatory cell death pathways (35). Furthermore, subtilisin-like serine proteases termed phytaspases (plant aspartate-specific protease) have been shown to control cell death in plants $(36,37)$. Phytaspases exhibit caspase-like specificity and can induce immune-related cell death in response to viral infections (38). Our findings now suggest that both types of proteases (subtilisin-like and caspase-like) regulate gasdermin-dependent cell death in fungi, likely in the context of organismal defense. While metacaspases (39), distantly related to caspases, have been previously shown to control fungal cell death in various physiological and stress conditions (1), our work proposes an unprecedented role for caspase-like domains in fungal non-self recognition processes.

Beside the significance for the evolution of proteolytic control of RCD, our work uncovers that, as described in mammals, some gasdermin proteins are downstream of NOD-like receptors or NLR-like proteins. Nevertheless, it appears that in fungi the signaling pathways leading to activation of gasdermin cytotoxicity are less complex with the NLR protein carrying directly the protease domain, while in mammals the NLRs use adapter domains (i.e. CARD Caspase activation and recruitment domain) to activate downstream caspases directly (in some cases) or though adaptor proteins like ASC (Apoptosis-associated speck-like protein containing a CARD) (Fig. 4E) (40).

\section{Allorecognition systems in fungi: branching out of broader and unexplored cell death pathways}

Our findings suggest that the het-Q1/het-Q2 allorecognition system found in P. anserina has emerged from an ancestral het-Q1/het-Q2-like two-gene cluster. In this model, genomic rearrangements have resulted in the het-Q2 gene, which encodes only the protease domain of a HET-Q2-like protein and hence results in a constitutively active protease, triggering cell death when co-expressed with HET-Q1. In this hypothesis, the het-Q1/het-Q2 system would represent an evolutionary exaptation by which a preexisting immune pathway has been refurbished into an allorecognition system (41). A similar evolutionary scenario has been proposed for a two gene cluster involving a NLR and a different pore-forming toxin (HET-S) in Podospora, where a point mutation and a transposon insertion have generated an incompatibility system (het-s/het$S$ allelic system) (42). In addition, several other NLR-based allorecognition systems are 
considered to originate from broader non-self recognition-dedicated molecular pathways (43). The exaptation model could equally be proposed for the $r c d-1-1 / r c d-1-2$ allorecognition system in N. crassa, where genomic rearrangements have been associated with the rcd-1 locus (22). Our study supports thus further the hypothesis that allorecognition genes originate from preexisting broader signaling pathways, likely involved in xenorecognition and mediation of interspecific biotic interactions (44).

\section{Conclusion}

The present results establish that in filamentous fungi, in addition to necroptotic-like pathways regulated by amyloid signaling (5), gasdermin-dependent pathways regulated by proteolytic cleavage abound. Mammals and fungi thus share core components and molecular mechanisms of RCD pathways. We hereby critically expand the evolutionary parallels between RCD pathways in two of the major clades of Opisthokonta (fungi and animals). While the extent of the molecular and functional similarities (and differences) remain to be fully explored, our study unveils a remarkable evolutionary conservation in cell death signaling and execution in the context of eukaryotic innate immunity and as such represents a further step towards the molecular definition of the fungal immune system.

\section{Methods and materials}

\section{Strains and plasmids}

Podospora anserina strains used in this study were wild type het-Q1 and het-Q2 and the $\Delta$ het-Q1 ( 4 het-Q) strain. All strains were cultivated at $26^{\circ} \mathrm{C}$ using standard procedures. To produce the $\Delta$ het- $Q$ strain, we replaced the het-Q1 ORF (Pa_7_10775) with the nat1 gene, encoding a nourseothricin acetyl transferase, procuring resistance to the nourseothricin antibiotic. The het-Q1 deletion cassette consists of the nat1 gene flanked by the upstream and downstream regions of the het-Q1 gene. To produce the deletion cassette, we amplified $1055 \mathrm{bp}$ upstream of het-Q1 ORF using primers 5' AATATTGGAGAAGCAAAATGAGGC 3' and 5' TTTAGGTTCTTCTGAAAAGAGATAC 3' and cloned the PCR fragment upstream of the nat1 gene. Downstream of the nat1 gene, we cloned a PCR fragment corresponding to $894 \mathrm{bp}$ downstream of Pa_7_10775, starting from the stop codon of the gene. The PCR fragment was amplified with the primers 5' GAGGAAGGTTATAATCGATGTGTG 3' and 5' GCAGTGGCGTTTGTCTTCGC 3'. The het-Q1 deletion cassette was constructed into a pBlueScript II-derived vector (termed 'p1'). To delete the het-Q1 gene, we used $5 \mu \mathrm{g}$ of $2.76 \mathrm{kbp} \mathrm{NcoI-linearized} \mathrm{10775::nat1} \mathrm{(deletion} \mathrm{cassette)}$ DNA fragment, which we introduced into a het-Q1 $\triangle P a K u 70$ strain. Nourseothricin-resistant transformants were selected, PCR screened and backcrossed with the wild type reference strain to obtain $\Delta$ het- $Q$ homokaryons. The barrage phenotype segregated with the nourseothricin- 
resistance phenotype in the progeny with resistant transformants unable to produce a barrage reaction with a het-Q2 strain.

The het-Q1 gene was amplified with primers 5' GTGTTTCGCTTGTTCAATCCG 3' and 5' TTTTCACAATGGAAATCGGGATG 3' and the $1.85 \mathrm{kbp}$ PCR fragment was cloned with EcoRV into a pBlueScript-derived vector carrying the hygromycin resistance $h p h$ gene. Similarly, we amplified the het-Q2 gene using primers 5' ACGATCAAACAACCCAGTTCGG 3' and 5' GAACCCCGATTTCAAGTATATGC 3' and the PCR fragment (2.55 kbp) was cloned with the EcoRV restriction enzyme into the SKhph vector. The final constructs were sequenced and the vectors (SKhph, SKhph-Q1, SKhph-Q2) were used for viability reduction essays.

For heterologous expression of het-Q1 and het-Q2 in the yeast Saccharomyces cerevisiae, the CDS (coding sequences) of the two genes were PCR amplified and cloned in the pRS413 (45) and pGal vectors, respectively. The het-Q1 sequence was amplified with primers 5' TTGGATCCATGCCCACCAAAACCTCCCAAC 3' and 5' GGTCGACTCACGCCTTGGCAGCAACATC 3', carrying BamHI and SalI restriction sites. The $853 \mathrm{bp}$ PCR fragment was cloned in the pRS413 vector digested with BamHI and XhoI, to obtain the pRS413-het-Q1 plasmid. The het-Q1 sequence was under the control of the strong constitutive promoter of the gpd (glyceraldehyde3-phosphate dehydrogenase) gene. The het-Q2 coding sequence was cloned under the control of a galactose-inducible promoter (gal1), using 'gap repair' methods (46), to produce the pGal-hetQ2 plasmid.

Site-directed mutagenesis was performed with QuikChange II kit (Agilent), using manufacturer recommended procedures.

\section{Mapping and positional cloning of het-Q1 and het-Q2}

To identify the het-Q locus and clone the het-Q1 and het-Q2 genes, we first undertook a classical genetics mapping to situate more precisely the locus on chromosome 7. For this we crossed a P. anserina het-Q2 strain with a het-Q1 $\triangle P a A T G 1$ strain (PaATG1::hph), where the PaATG1 is replaced with hygromycin resistance cassette. The PaATG1 gene deletion has been previously described and characterized (25). We isolated 112 uninucleated spores, from which 78 spores germinated. Hygromycin-resistance screen showed that 35 (45\%) spores are resistant to the antibiotic, while 43 (55\%) are sensitive. Barrage tests (with het-Q1 and het-Q2 tester strains) further showed that only 2 recombinant spores (het-Q2 $\triangle P a A T G 1$ ) have been obtained from the 78 germinated spores. Based on the $2.5 \%$ of recombination between the PaATG1 and the het-Q1 loci, and knowing that $1 \mathrm{cM}$ equals $\sim 25 \mathrm{kbp}$ in P. anserina, we calculated that the physical distance between the two loci is $\sim 65 \mathrm{kbp}$. 
To pinpoint the het- $Q$ locus, we undertook a 'chromosomal walking' approach (47) with cosmids and plasmids carrying DNA from the het-Q1 strain, previously used to sequence $P$. anserina reference genome (S genotype) (48). We used four cosmids (GA0AAD19ZF04 (CQ2), GA0AAD26ZD03 (CQ3), GA0AAD29ZA05 (CQ4) and GA0AAD50ZA08 (CQ5)) and five plasmids (GA0AB158BG07 (Q1), GA0AB263AH07 (Q2), GA0AB26BB11 (Q3), GA0AB218BA11 (Q4) and GA0AB91DC06 (Q5)) encompassing a 200-kbp region around the PaATG1 (Pa_7_10890) locus. Cosmids were carrying the $h p h$ gene (hygromycin resistance) and were transformed in a het-Q2 strain and viable transformants quantified. Transformations were performed with $10 \mu \mathrm{g}$ of cosmid DNA. Plasmids used for the identification of the het- $Q$ locus were used in cotransformation with the $h p h$-bearing vector pCSN43 (GenBank: LT726869.1) (3:1 ratio for 12 $\mu \mathrm{g}$ total DNA). These viability reduction assays identified the plasmid GA0AAD29ZA05 (CQ4), carrying an 11-kbp insert and containing 5 genes, as the region, in which het-Q1 is situated. Five variable in size deletions were produced and after viability reduction tests, it was determined that vectors carrying DNA fragments containing the Pa_7_10775 gene reduced the number of hygromycin-resistant transformants.

\section{Viability reduction and vegetative incompatibility essays}

Vegetative incompatibility essays (barrage tests) were performed on standard corn meal agar DO medium. Viability essays were performed by transformation of protoplasts (or spheroplasts) of P. anserina strains (49)(of the het-Q1, het-Q2 or $\Delta$ het-Q genotypes) with the SKhph, SKhph-Q1 and SKhph-Q2 vectors carrying the $h p h$ gene, which procures hygromycin resistance. Transformations were carried with 7.5 or $10 \mu \mathrm{g}$ of vector DNA. Transformants were counted after 6 days of incubation at $26^{\circ} \mathrm{C}$.

\section{Saccharomyces cerevisiae spot test essay}

The pGal-het-Q2, pRS413-het-Q1 and the two vectors (pGal and pRS413) expressing free GFP (Green Fluorescent Protein) were transformed or co-transformed in a S. cerevisiae (mat-a, his3 $\Delta 1$, leu2 $\Delta 0$, ura3 $\Delta 0$, trp1::LEU2) using the One-step transformation protocol (50). Tryptophan and histidine prototrophic transformants were selected and grown in liquid media $(10 \%$ glucose $)$. Culture suspensions $\left(1.5 .10^{7}\right.$ cellules $\left./ \mathrm{ml}\right)$ were serially diluted (by a factor of $10)$ and $5 \mu \mathrm{l}$ of each suspension were spotted on synthetic media with galactose $(2 \%)$ or glucose $(2 \%)$ as carbon source. Transformants were incubated at $30^{\circ} \mathrm{C}$ from 3 to 5 days.

\section{Crude cell extracts and in vitro proteolytic cleavage of HET-Q1}

Protoplasts of different P. anserina strains were prepared using standard methods (51) at a final concentration of $10^{6}$ protoplasts $/ \mu$ l. Cell extracts were prepared from $200 \mu \mathrm{l}$ of 
protoplasts, which were suspended in $10 \mathrm{mM}$ phosphate buffer $\left(\mathrm{Na}_{2} \mathrm{HPO}_{4}, \mathrm{NaH}_{2} \mathrm{PO}_{4}\right) \mathrm{pH} 7.4$

498 after centrifugation at $4^{\circ} \mathrm{C}, 5000 \mathrm{rpm}$ for $10 \mathrm{~min}$. Protoplast suspensions were sonicated (at

499 amplitude four with a Soniprep 150) twice for 10s with 30s on ice between sonication cycles.

500 Sonicated samples were centrifuged at $4^{\circ} \mathrm{C}, 10000 \mathrm{rpm}$ for $10 \mathrm{~min}$ and $160 \mu \mathrm{l}$ of supernatant

501 were transferred to a new Eppendorf tube and kept at $4^{\circ} \mathrm{C}$.

502 Experiments were carried by mixing $50 \mu \mathrm{l}$ of each crude extract and incubating the 503 reaction mixtures at $26^{\circ} \mathrm{C}$ up to an hour. To stop the reaction at a specific time-point, $15 \mu \mathrm{l}$ of the 504 crude cell extract mixtures were added to $15 \mu \mathrm{l}$ of 3x protein loading dye (SDS 3.4\%).

\section{Data Availability}

All data from this work are included in the main text and the SI Appendix of the paper.

507

508

509

510

511

512

513

514

515

516 
A.



het-Q2



het-Q2

\section{$\Delta$ het-Q} het-Q1

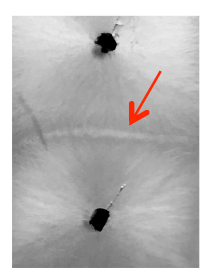

het-Q2
$\Delta$ het-Q het-Q2

C.

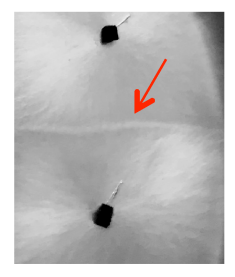

het-Q1
B.

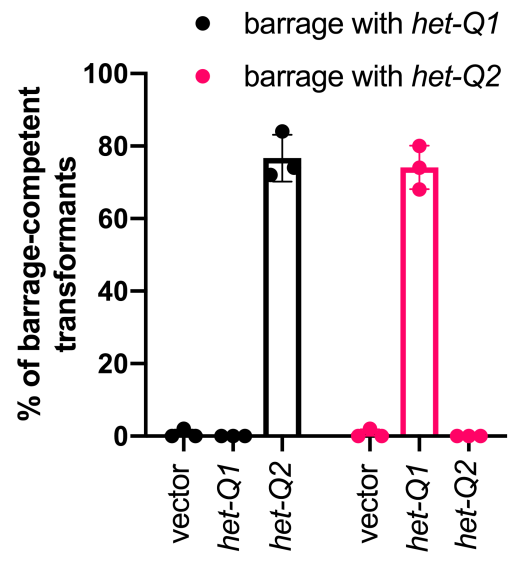

Nothing detected $20 \% \quad 30 \% \quad 40 \% \quad 50 \% \quad 60 \% \quad 70 \% \quad 80 \% \quad 90 \% \quad 100 \%$
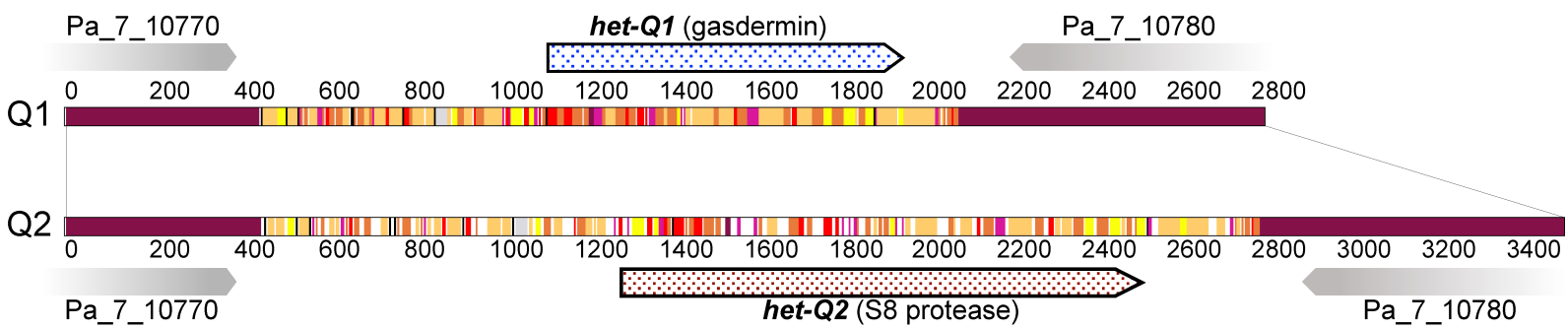

534 Figure 1. Gene idiomorphs het-Q1 (gasdermin homolog) and het-Q2 (S8 serine protease) define an RCD system in the context of heterokaryon incompatibility (allorecognition) in

Podospora anserina. A. Cell death between P. anserina strains of antagonistic het-Q1 and het-Q2 genotypes manifest with the appearance of 'barrage' reaction between the fungal colonies (red arrows). Deletion of the het-Q1 gene (Pa_7_10775) produces a $\Delta$ het- $Q$ strain (we term it $\Delta$ het-Q as the het-Q1 and het-Q2 strains are isogenic with the exception of the het-Q locus) and abolishes the cell death reaction (barrage formation) with a het-Q2 strain (black arrow). Reintroduction of het-Q1 in a $\Delta$ het- $Q$ strain restores the barrage reaction with a het-Q2 tester strain, while introduction of het$Q 2$ in the $\Delta$ het- $Q$ background procures the ability to the transformants to produce a barrage reaction with a het-Q1 tester strain. B. Quantification of het-Q1/het-Q2 cell death, measuring the percentage of transformants producing barrage reaction with a het-Q1 and a het-Q2 tester strains. Experiments were performed in triplicate with 50 transformants tested per experiment. Empty vector has been used as a negative control. C. Graphic representation of the het- $Q$ locus as found in strains from the het-Q1 (Q1) and the het-Q2 genotypes (Q2). Shown is an alignment of the het-Q locus from a het-Q1 and het-Q2 strain. Sequence similarity at nucleotide level is color-coded. Regions encoding the idiomorphic genes show low sequence similarity (in 40-60\% range), while regions encoding the neighbouring genes (Pa_7_10770 and Pa_7_10780) show sequence similarity above 90\% between the two genotypes. 
A.

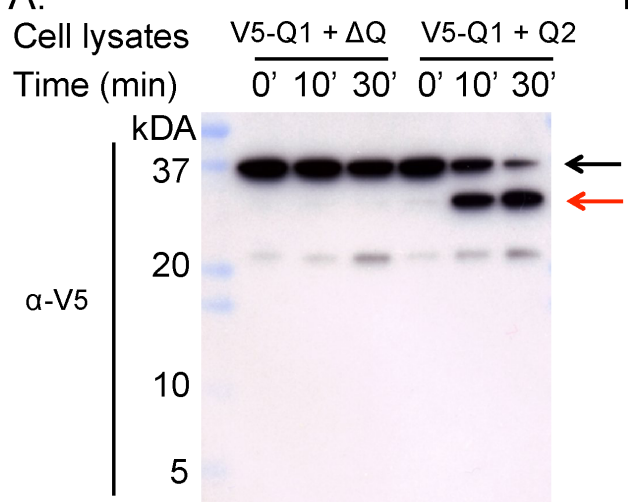

D.

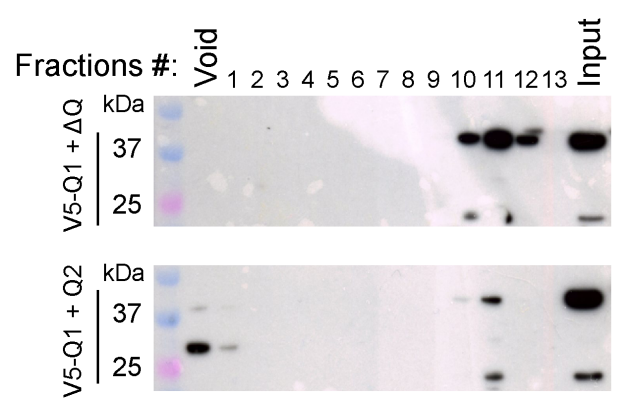

B.

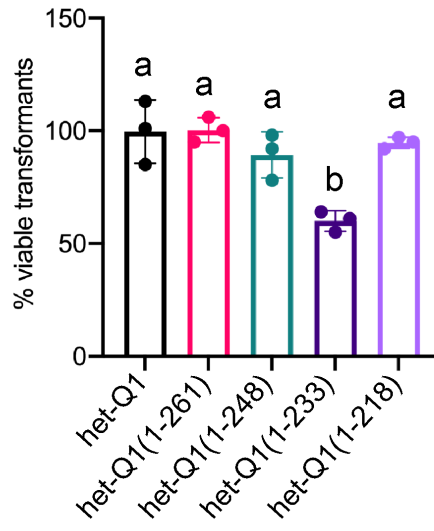

E.

$\mathrm{F}$.

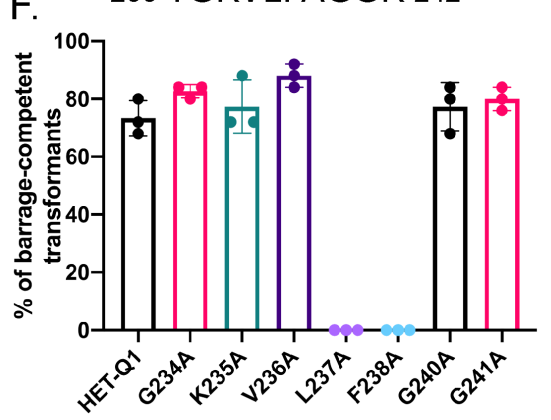

C.

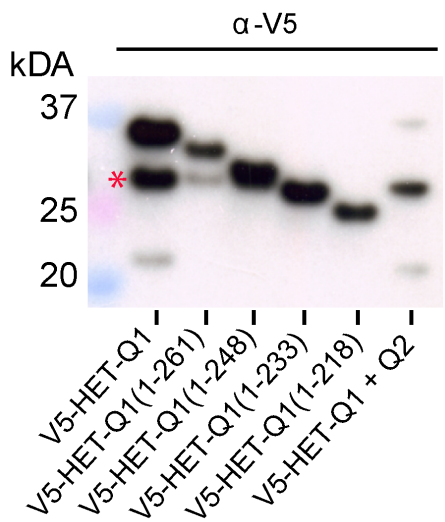

G.

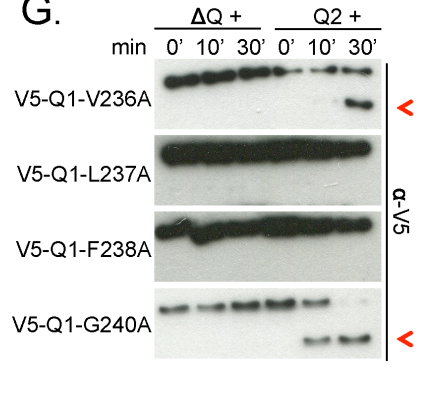

Figure 2. HET-Q2-dependent proteolytic cleavage of the gasdermin-like protein HET-Q1

induces cell death in P. anserina. A. Western blot showing accumulation of processed V5-HET-Q1 (V5-Q1) in a het-Q2-dependent manner. Crude cell lysates from V5-HET-Q1-expressing strain were mixed and incubated (for up to $30 \mathrm{~min}$ ) with cell extracts from a $\Delta$ het-Q strain (V5-Q1 $+\Delta \mathrm{Q}$ ) or from a het-Q2 strain (V5-Q1 + Q2). In the first reaction (V5-Q1 + $\Delta \mathrm{Q})$, V5-HET-Q1 remained unprocessed and appeared as single band at the expected size for the full-length protein ( $35-\mathrm{kDa})$ (black arrow). However, when mixed with extracts from a het-Q2 strain (V5-Q1 + Q2), a smaller V5-HETQ1 fragment (27-30-kDa) (red arrow) appears and accumulates in time, while the amount of fulllength protein progressively decreases. B. Quantification of transformation viability of $\Delta$ het-Q transformants with truncated het-Q1 alleles. Four truncations of het-Q1 were introduced into $\Delta$ het-Q protoplasts and number of viable transformants counted. Three of the truncations (het-Q1(1-261), het-Q1(1-248) and het-Q1(1-218)) produced wild-type levels of viable transformants. Transformations with the fourth truncation - het-Q1(1-233) - resulted in $\sim 45 \%$ reduction of viable transformants. Experiments were performed in triplicate. P-value $(a \neq b)<0.01$, one-way ANOVA with Tukey's multiple comparisons test. C. Western blot of full-length and truncated V5-HET-Q1 variants. Cytotoxic V5-HET-Q1(1-233) is of similar molecular size than processed V5-HET-Q1 in mixtures of cell extracts (V5-HET-Q1 + Q2). Protein bands corresponding to unspecific proteolysis due to high levels of protein expression are shown with red asterisk. D. Polyacrylamide gel electrophoresis (PAGE) and Western blot of FPLC (Fast protein liquid chromatography) fractions of crude cell extracts mixtures. Cell extracts containing V5-HET-Q1 were mixed with cell extracts from a $\Delta$ het-Q strain (V5-Q1 $+\Delta \mathrm{Q}$ ) (top panel) or from a het-Q2 strain (V5-Q1 + Q2) (bottom panel). 
Processed V5-HET-Q1 in mixtures containing the HET-Q2 protease elutes in the void volume, containing protein aggregates of high molecular weight (above 1-MDa), while unprocessed V5-HETQ1 elutes in fractions corresponding to proteins with lower molecular weight, close to that of monomeric V5-HET-Q1 ( 35-kDa). E. Protein sequence of HET-Q1(232-243). Amino acid residues substituted with alanine residues are marked with asterisks. Black asterisks indicate residues, which substitution did not impact the cell death reaction. Red asterisk indicates that the substitution abolishes the cell death reaction and yellow asterisk indicates that it impacts partially cell death. F. Quantification of barrage-inducing transformants expressing mutant V5-HET-Q1 variants. Experiments were performed in triplicate with 25 tested transformants per experiment. Mutant variants HET-Q1(L237A) and HET-Q1(F238A) are significantly affected in their cell death-inducing ability (P-value < 0.0001, one-way ANOVA with Tukey's multiple comparisons test). G. Western blots of V5-HETQ1 mutant variants in cell extract mixtures containing HET-Q2 protease (Q2 +) or without $(\Delta \mathrm{Q}+)$. Mutations affecting the cell death reaction (V5-Q1-L237A and V5-Q1-F238A) prevent proteolytic cleavage of HET-Q1. Proteolytically processed mutants (V5-Q1-V236A and V5-Q1-G240A) are shown with red arrowheads.

A.

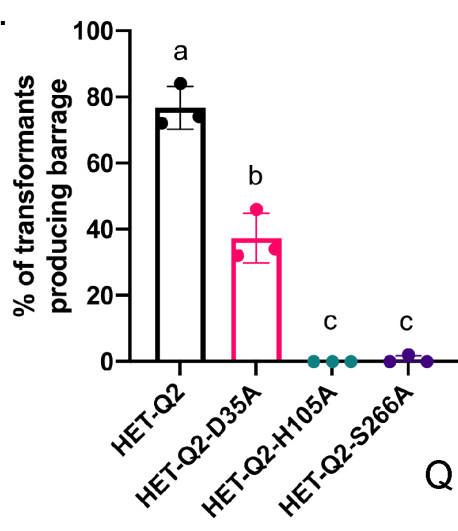

B.

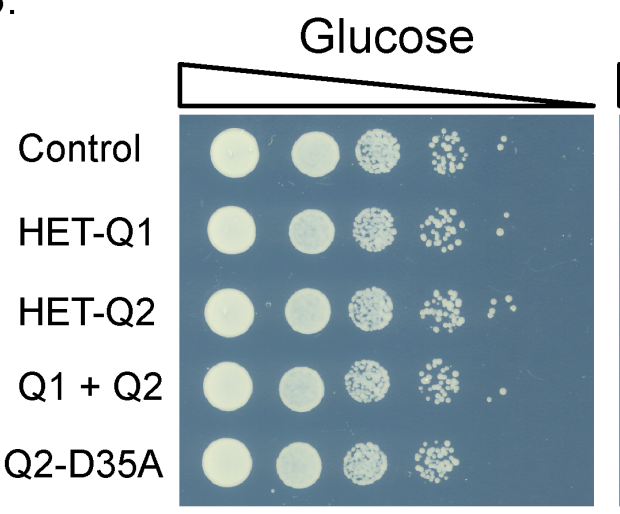

Galactose

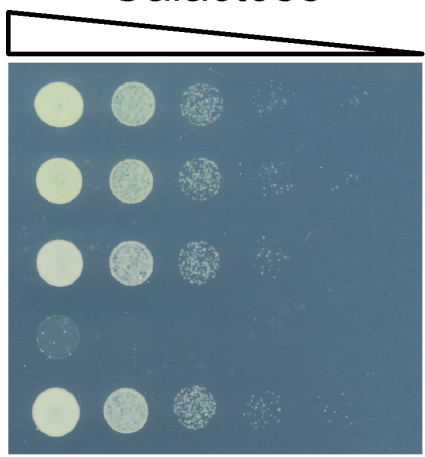

Figure 3. Proteolytic activity of the subtilisin-like protease HET-Q2 is essential for gasdermin cytotoxicity. A. Quantification of cell death (barrage) induction by transformants expressing HETQ2 and mutant HET-Q2 variants. Alanine substitutions of residues forming the predicted catalytic triad of HET-Q2 (D35A, H105A and S266A) affect the cell death reaction. Experiments were performed in triplicate with 50 transformants per experiment. P-value $(a \neq b \neq c)<0.0001$, one-way ANOVA with Tukey's multiple comparisons test. B. Saccharomyces cerevisiae spot test essay on repressed (glucose) and inducible (galactose) growth media. Co-expression of HET-Q1 with HET-Q2 (Q1 + Q2) leads to growth arrest of S. cerevisiae, while individual expression of both proteins or co- 
601

602

603

expression of the gasdermin with a catalytically affected protease variant (HET-Q2-D35A) did not affect yeast growth.

A.
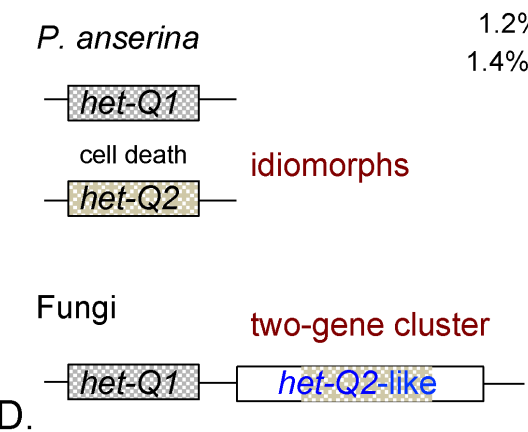

D.

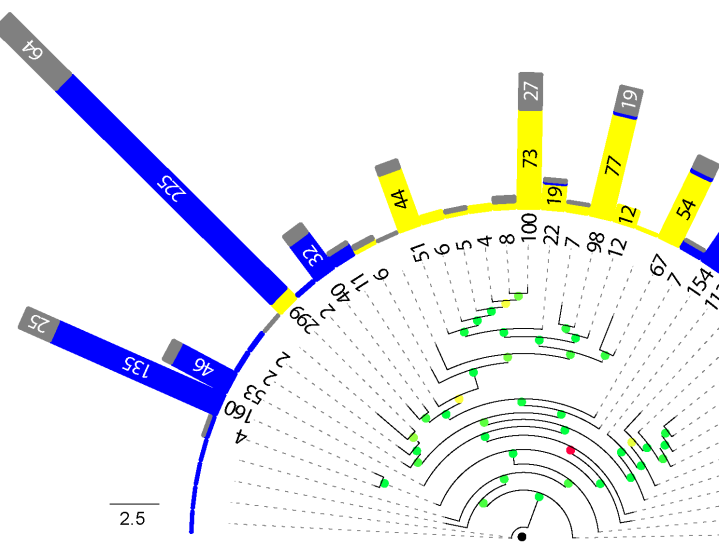

B.
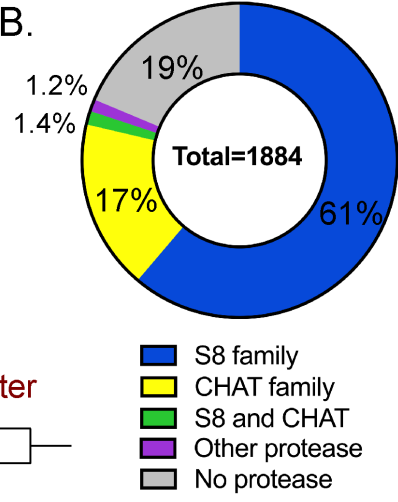

C.

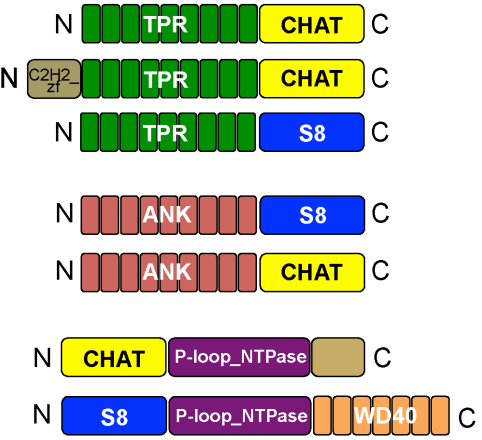

$221(63 \%)$

$19(5 \%)$

$59(5 \%)$

$37(3 \%)$

$9(3 \%)$

48 (14\%)

E.

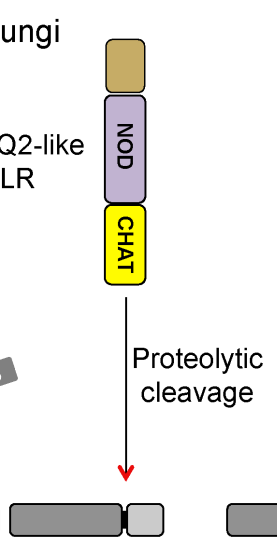

$28(2 \%)$

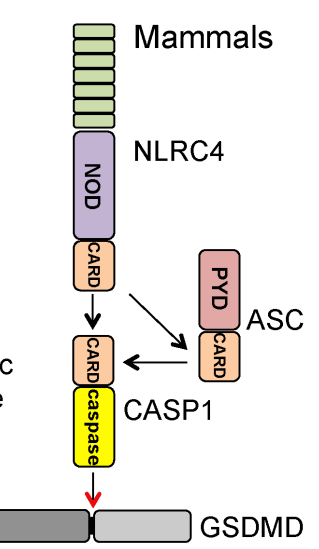

605

606

607

608

609

610

611

612

613

614

615

616

617

618

619

620

Figure 4. Gasdermin het-Q1 homologs are genomically clustered with protease-encoding het-

Q2-like genes in filamentous fungi. A. Cartoon representation of het-Q1/het-Q2 gene idiomorphs, defining the allorecognition cell death reaction in P. anserina and of het-Q1/het-Q2-like gene clusters, distributed broadly in Fungi. B. Percentage of gasdermin homologs situated in the vicinity (10 kbp upstream and downstream) of protease-encoding het-Q2-like genes. C. Cartoons representing annotated HET-Q2-like protein architectures. Given are the numbers of proteins in each category and the percentage that each category represents from the total number of CHAT- or S8-containing HET-Q2-like proteins. D. Maximum-likelihood phylogenetic tree of het-Q1 genes. Numbers of het-Q1 homologs in collapsed branches are shown for each branch. Branch support is color-coded: red -0.00 , yellow -0.50 , grass-like green -0.75 , green -1 . Coloured bars at tip of branches show the type of protease domain found on the clustered HET-Q2-like proteins (blue - S8 family, yellow - caspase-like CHAT family) or the number of het-Q1 homologs not clustered with a het-Q2-like gene (grey). E. Comparative models of cell death pathways in Fungi and Mammals. Shown is an example of a CHAT-domain containing fungal NLR-like protein - a HET-Q2-like protein - hypothesized to activate through proteolytic cleavage a downstream HET-Q1 protein. Such HETQ2-like/HET-Q1 pathways appear analogous to pyroptotic-inducing cell death pathways in 
621

622

623

624

625

626

627

628

629

630

631

632

633

634

635

636

637

638

639

640

641

642

643

644

645

646

647

648

649

650

mammals. Given example shows the downstream activation of GSDMD (gasdermin D) by NLRC4 (NLR family CARD-containing protein), which can directly activate caspase-1 (CASP1) or indirectly via the ASC protein (Apoptosis-associated speck-like protein containing a CARD) (40). NOD Nucleotide-binding and oligomerization domain; CARD - Caspase-activation and recruitment domain; PYD - Pyrin domain.

\section{Acknowledgments}

The work was funded by recurrent funds from CNRS and University of Bordeaux to S.J.S. and an ANR grant to S.J.S. (ANR SFAS AAP CE11). WD was supported in part by the National Science Centre of Poland (grant no. 2015/17/D/ST6/04054). We thank Robert Debuchy for providing the cosmids and plasmids for the cloning of het-Q1.

\section{Author contributions}

C.C., S.J.S. and A.D. designed research; C.C., W.D., A.G.F., B.P. and A.D. performed research; C.C., W.D., S.J.S., and A.D. analyzed data; and A.D. wrote the paper

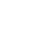

(1)


1. Gonçalves AP, Heller J, Daskalov A, Videira A, Glass NL (2017) Regulated forms of cell death in fungi. Front Microbiol 8:1837.

2. Saupe SJ (2000) Molecular genetics of heterokaryon incompatibility in filamentous ascomycetes. Microbiol Mol Biol Rev 64(3):489-502.

3. Zhang D-X, Nuss DL (2016) Engineering super mycovirus donor strains of chestnut blight fungus by systematic disruption of multilocus vic genes. Proc Natl Acad Sci USA 113(8):2062-2067.

659

660

661

662

663

664

665

666

667

668

4. Debets F, Yang X, Griffiths AJF (1994) Vegetative incompatibility in Neurospora: its effect on horizontal transfer of mitochondrial plasmids and senescence in natural populations. Curr Genet 26(2):113-119.

5. Daskalov A, et al. (2016) Identification of a novel cell death-inducing domain reveals that fungal amyloid-controlled programmed cell death is related to necroptosis. Proc Natl Acad Sci USA 113(10):2720-2725.

6. Daskalov A, Mitchell PS, Sandstrom A, Vance RE, Glass NL (2020) Molecular characterization of a fungal gasdermin-like protein. Proc Natl Acad Sci USA.

7. Salvesen GS, Hempel A, Coll NS (2016) Protease signaling in animal and plant-regulated cell death. FEBS J 283(14):2577-2598.

8. Julien O, Wells JA (2017) Caspases and their substrates. Cell Death Differ 24(8):1380-

9. Chang HY, Yang X (2000) Proteases for cell suicide: functions and regulation of caspases.

10. LaRock CN, Cookson BT (2013) Burning down the house: cellular actions during pyroptosis. PLoS Pathog 9(12):e1003793.

11. Shi J, Gao W, Shao F (2017) Pyroptosis: Gasdermin-Mediated Programmed Necrotic Cell Death. Trends Biochem Sci 42(4):245-254.

12. Shi J, et al. (2015) Cleavage of GSDMD by inflammatory caspases determines pyroptotic cell death. Nature 526(7575):660-665.

13. Kayagaki N, et al. (2015) Caspase-11 cleaves gasdermin D for non-canonical inflammasome signalling. Nature 526(7575):666-671.

14. Aglietti RA, et al. (2016) GsdmD p30 elicited by caspase-11 during pyroptosis forms pores in membranes. Proc Natl Acad Sci USA 113(28):7858-7863.

15. Liu X, et al. (2016) Inflammasome-activated gasdermin D causes pyroptosis by forming

16. Ding J, et al. (2016) Pore-forming activity and structural autoinhibition of the gasdermin

17. Sborgi L, et al. (2016) GSDMD membrane pore formation constitutes the mechanism of

18. Broz P, Pelegrín P, Shao F (2020) The gasdermins, a protein family executing cell death

19. Rogers C, et al. (2017) Cleavage of DFNA5 by caspase-3 during apoptosis mediates progression to secondary necrotic/pyroptotic cell death. Nat Commun 8:14128. 
20. Zhou Z, et al. (2020) Granzyme A from cytotoxic lymphocytes cleaves GSDMB to trigger pyroptosis in target cells. Science 368(6494).

21. Wang Y, et al. (2017) Chemotherapy drugs induce pyroptosis through caspase-3 cleavage of a gasdermin. Nature 547(7661):99-103.

22. Daskalov A, Gladieux P, Heller J, Glass NL (2019) Programmed Cell Death in Neurospora crassa Is Controlled by the Allorecognition Determinant rcd-1. Genetics 213(4):13871400.

23. Paoletti M (2016) Vegetative incompatibility in fungi: From recognition to cell death, whatever does the trick. Fungal Biol Rev 30(4):152-162.

24. Esser K (1974) in Bacteria, bacteriophages, and fungi, ed King RC (Springer US, Boston, MA), pp 531-551.

25. Pinan-Lucarré B, Balguerie A, Clavé C (2005) Accelerated cell death in Podospora autophagy mutants. Eukaryotic Cell 4(11):1765-1774.

26. Glass NL, Grotelueschen J, Metzenberg RL (1990) Neurospora crassa A mating-type region. Proc Natl Acad Sci USA 87(13):4912-4916.

27. Söding J, Biegert A, Lupas AN (2005) The HHpred interactive server for protein homology detection and structure prediction. Nucleic Acids Res 33(Web Server issue):W244-8.

28. Hohl M, Stintzi A, Schaller A (2017) A novel subtilase inhibitor in plants shows structural and functional similarities to protease propeptides. J Biol Chem 292(15):6389-6401.

29. Carter P, Wells JA (1988) Dissecting the catalytic triad of a serine protease. Nature 332(6164):564-568.

30. Daskalov A, et al. (2015) Signal transduction by a fungal NOD-like receptor based on propagation of a prion amyloid fold. PLoS Biol 13(2):e1002059.

31. Aravind L, Koonin EV (2002) Classification of the caspase-hemoglobinase fold: detection of new families and implications for the origin of the eukaryotic separins. Proteins $46(4): 355-367$.

32. Jernigan KK, Bordenstein SR (2015) Tandem-repeat protein domains across the tree of life. PeerJ 3:e732.

33. Daskalov A, Paoletti M, Ness F, Saupe SJ (2012) Genomic clustering and homology between HET-S and the NWD2 STAND protein in various fungal genomes. PLoS One $7(4): \mathrm{e} 34854$.

34. Kovacs SB, Miao EA (2017) Gasdermins: effectors of pyroptosis. Trends Cell Biol 27(9):673-684.

35. Heutinck KM, ten Berge IJM, Hack CE, Hamann J, Rowshani AT (2010) Serine proteases of the human immune system in health and disease. Mol Immunol 47(11-12):1943-1955.

36. Chichkova NV, et al. (2010) Phytaspase, a relocalisable cell death promoting plant protease with caspase specificity. EMBO J 29(6):1149-1161.

37. Reichardt S, et al. (2018) The tomato subtilase family includes several cell death-related proteinases with caspase specificity. Sci Rep 8(1):10531.

38. Figueiredo J, Sousa Silva M, Figueiredo A (2018) Subtilisin-like proteases in plant defence: the past, the present and beyond. Mol Plant Pathol 19(4):1017-1028.

39. Tsiatsiani L, et al. (2011) Metacaspases. Cell Death Differ 18(8):1279-1288. 
40. Andrade WA, Zamboni DS (2020) NLRC4 biology in immunity and inflammation. J Leukoc Biol 108(4):1117-1127.

41. Gould SJ, Vrba ES (1982) Exaptation-a Missing Term in the Science of Form. Paleobiology 8(01):4-15.

42. Daskalov A, Saupe SJ (2015) As a toxin dies a prion comes to life: A tentative natural history of the [Het-s] prion. Prion 9(3):184-189.

43. Daskalov A, Dyrka W, Saupe SJ (2020) in Genetics and Biotechnology, eds Benz JP, Schipper $\mathrm{K}$ (Springer International Publishing, Cham), pp 123-141.

44. Paoletti M, Saupe SJ (2009) Fungal incompatibility: evolutionary origin in pathogen defense? Bioessays 31(11):1201-1210.

45. Sikorski RS, Hieter P (1989) A system of shuttle vectors and yeast host strains designed for efficient manipulation of DNA in Saccharomyces cerevisiae. Genetics 122(1):19-27.

46. Oldenburg KR, Vo KT, Michaelis S, Paddon C (1997) Recombination-mediated PCRdirected plasmid construction in vivo in yeast. Nucleic Acids Res 25(2):451-452.

47. Bender W, Spierer P, Hogness DS (1983) Chromosomal walking and jumping to isolate DNA from the Ace and rosy loci and the bithorax complex in Drosophila melanogaster. $J$ Mol Biol 168(1):17-33.

48. Espagne E, et al. (2008) The genome sequence of the model ascomycete fungus Podospora anserina. Genome Biol 9(5):R77.

49. Brygoo Y, Debuchy R (1985) Transformation by integration in Podospora anserina. Molec Gen Genet 200(1):128.

50. Chen DC, Yang BC, Kuo TT (1992) One-step transformation of yeast in stationary phase. Curr Genet 21(1):83-84.

51. Ferrer S, Ramón D, Salom J, Vicente E, Uruburu F (1985) Protoplasts fromPodospora anserina: Isolation, purification, and transformation. Curr Microbiol 12(5):301-306. 